\title{
Interpretation of the contraindications to whooping cough vaccination
}

\author{
DAVID HULL
}

\begin{abstract}
The official recommendations on the contraindications to whooping cough immunisation are currently couched in general terms. The results of a simple inquiry made to directors of public health laboratories, general paediatricians, paediatric neurologists, medical officers, general practitioners, and health visitors to see how they would interpret the contraindications in certain specific cases suggest that there is considerable uncertainty on how the contraindications should be interpreted. This is probably one factor responsible for the fall in the rate of whooping cough immunisation in the United Kingdom.
\end{abstract}

\section{Introduction}

A recent national survey ${ }^{1}$ showed that after immunisation against whooping cough an infant has a slight but significant increased risk of developing an illness that results in brain damage. Neither this study nor case histories sufficiently persuasive on clinical criteria to justify compensation ${ }^{2}$ establish whether the whooping cough immunisation causes or precipitates the braindamaging condition. In some instances the temporal association will be by chance. Although there was little or no evidence that any one infant is more at risk than another of having such a reaction, it was thought prudent some years ago to recommend that infants should not be immunised if on general principles

\footnotetext{
Department of Child Health, University Hospital, Nottingham NG7 2UH

DAVID HULL, FRCP, DCH, professor
}

they might be thought to be predisposed to have a central nervous system reaction.

The current DHSS list of contraindications is as follows: "History of seizures, convulsions, or cerebral irritation in the neonatal period; history or family history of epilepsy or other diseases of the central nervous system in first-degree relatives; children with developmental neurological defects; any febrile illness, particularly respiratory, until the patient has fully recovered, or any severe local or general reaction to a preceding dose."

A personal or family history of allergy was once regarded as a contraindication to immunisation, but there is now a substantial body of medical opinion that no longer considers this to be so. Earlier statements did not include the limitation "in first-degree relatives." The contraindications on the manufacturer's information sheet differ slightly in detail.

I found these recommendations difficult to interpret and therefore turned to various practitioners and authorities to see what advice they would give to parents in a number of specific cases. Their answers reflected a wide difference in interpretation.

\section{Methods}

The respondents were asked whether, in a given clinical situation, they would advise against immunisation. One series of 10 questions (A) concerned information that would be on the discharge letter from the department of neonatal medicine, while the other series of 12 (B) concerned knowledge that would be gained during routine inquiry before the injection was given.

The respondents were 59 health visitors and 52 medical officers in the child health service working mainly in Nottingham or Sheffield; 25 general practitioners in Nottingham and South Wales; and 26 paediatricians (half of whom were professors), 11 paediatric neurologists, and 15 directors of public health laboratories working in many parts of the country.

Before considering the results, readers might be interested at this stage to consider their own answers to the 22 questions (table). 
Questions on contraindications to whooping cough. The respondents had to answer yes, no, or don't know

(A) Would you advise against whooping cough immunisation if you read in the discharge letter from the neonatal nursery/maternity unit:

(1) That the baby had been born prematurely and required artificial ventilation

(2) That the baby had had frequent apnoeic episodes

(3) That the baby had been born by difficult breech delivery and was jittery over the first 24 hours of life

(4) That the baby had been born by caesarean section and had required intubation and artificial ventilation for delay in onset of breathing

(5) That the baby had had twitching episodes when he was 4-days-old which responded to intravenous calcium and an alteration in his feeds

(6) That the baby had an haemangioma $1 \mathrm{~cm}$ in diameter on the left side of the face

(7) That the baby had a transient facial palsy due to forceps delivery

(8) That the baby had required phototherapy for three days and the bilirubin had risen above $300 \mathrm{mmol} / 1$ on the fourth day

(9) That the baby had been jittery after birth as a consequence of prolonged maternal sedation for pre-eclamptic toxaemia

(10) That the baby had had meningitis but responded well to treatment

(B) Would you advise against whooping cough immunisation if, after taking a full history from the parents, the only significant information was:

(1) That the baby had had "blue" attacks in the first three weeks of life

(2) That the baby was "mardy"-irritable, and awoke frequently in the night

(3) That the baby had had two breath holding attacks

(4) That the baby appeared to have a continuous nasal discharge

(5) That the baby had spina bifida, without weakness in the legs

(6) That the parents had had a previous child who reacted to DPT with a high fever

(7) That the father had had febrile convulsions as a child

(8) That the mother's sister was slow and required continuous medication for epilepsy

(9) That they had lost a previous child who died suddenly at home aged 6 months of age, not related to immunisation

(10) That the mother suffered poliomyelitis as a child and had got residual weakness in one arm

(11) That an older child had severe eczema as a child and had developed asthma, and that both parents themselves had an allergic tendency

(12) That the child had delayed motor development

\section{Results}

Figure 1 shows an analysis of all the answers to the questions for the various professional groups. Health visitors, medical officers, and general practitioners recommended against immunisation about twice as often as directors of public health laboratories and paediatricians. Paediatric neurologists fell between the two. In $21 \%$ of the cases health visitors as a group said that they did not know, and many said they would seek medical advice. Paediatricians, who are perhaps most familiar with the implications of the condition for the infants, decided

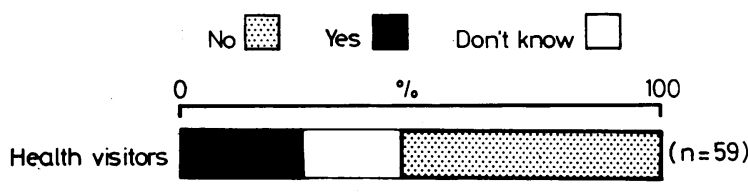

GPS

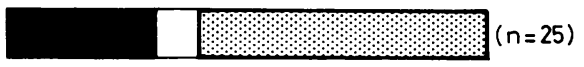

MOs

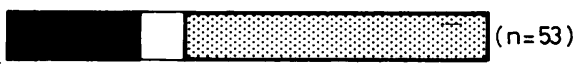

Paediatric neurologists

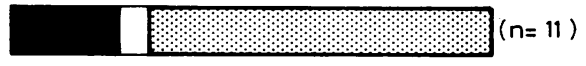

Paediatricians

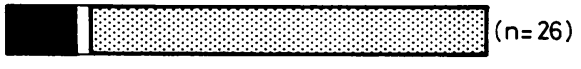

Directors

$\mathrm{PHL}$

$(n=15)$

FIG 1-Responses of health visitors, general practitioners, medical officers, paediatric neurologists, general paediatricians, and directors of public health laboratories (PHL) to all 22 questions in the table expressed as a percentage. one way or the other, but they were not consistent within the group. Several paediatricians and directors of public health laboratories considered that none of the conditions listed were contraindications to whooping cough immunisation.

As health visitors, medical officers, and general practitioners-that is, those who provide the immunisation service (the "givers") responded in a similar way, their answers to the individual questions were grouped together for simplicity of presentation and were compared with those of directors of public health laboratories and paediatricians - that is, those who usually give advice (the "advisers") -who were also grouped together (fig 2). About half the givers would

(a)
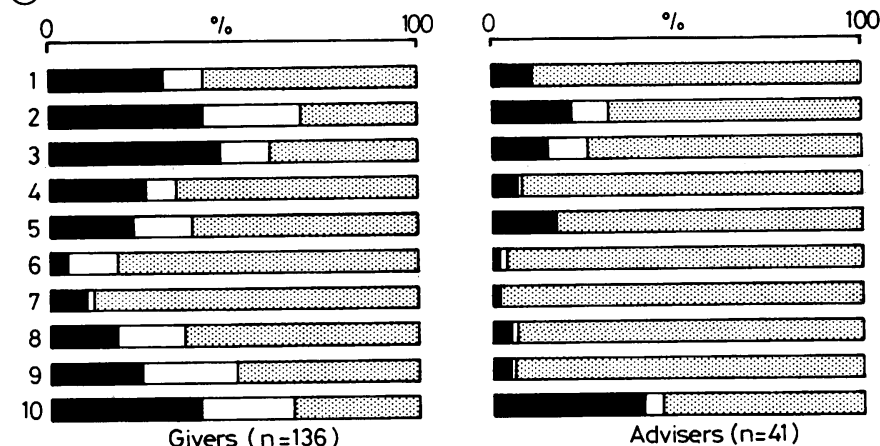

(b)

$$
\text { Givers }(n=136)
$$

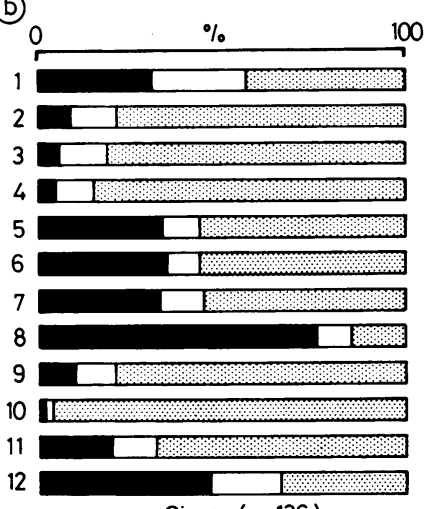

$\%$

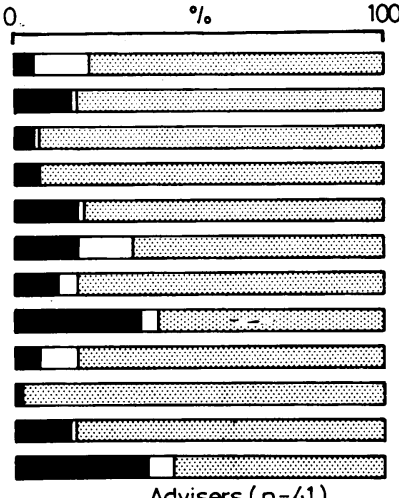

Givers $(n=136)$

Advisers $(n=41)$

unisation of a baby jittery after a breech delivery, the other half would not. Forty-seven per cent of the advisers would recommend against immunising a baby who had suffered from meningitis, whereas $53 \%$ would not. These figures illustrate that on many of these issues parents are likely to be given contradictory advice if they seek more than one opinion, and the confusion would not necessarily be resolved by seeking expert advice.

\section{Discussion}

A far more extensive and elaborate inquiry than this would be required to establish what professional advice parents were given in practice and a more sensitive one would be needed to find out what the parents understood from what they were told and how they reached a decision on the contraindications to whooping cough immunisation. This simple study, however, showed that there is often no consistent medical view. This confusion must be one factor contributing to the fall in the rate of whooping cough immunisation. The study also shows that in a wide range of cases some practitioners would be actively advising against immunisation and might even be refusing to give it.

If the original contraindications are interpreted literally most 
families would be included. Grandpa dying with a stroke, an aunt with schizophrenia, and a parent with a squint could all count. From this extreme there is a continuum of illness in the child or family history of disease that on general principles appears to have increasing relevance to the possibility of a damaging reaction to vaccine. The question is where to draw the line. One has the impression that the profession has been increasingly reluctant to recommend immunisation in the light of the extensive public debate on vaccine causing brain damage and the Vaccine Damage Payments Act. The decision by the givers about whether immunisation is contraindicated is bound to be influenced to a lesser or greater degree by the fear that immunisation, if recommended, might cause brain damage to the child they are trying to help. That is fear enough; to introduce at this point the added fear of the possible legal, professional, and personal consequences of immunising a child when some might consider that a contraindication was present (although in themselves very important considerations) does not add to this argument.

It could now be argued that the enforcement of some of the contraindications is against the interests of the individual. Although a child who has suffered some brain damage at birth may be at an increased risk of reacting unfavourably to the vaccine, he may be even more at risk if he contracts the disease itself. When the population at large is protected against the infection then the vulnerable individual may share that protection without taking the risk of the immunising procedure. In this instance all the contraindications are valid from the individual's point of view, but when little such protection exists, and that would appear to be the increasing situation now for whooping cough, implementing some of the contraindications runs against the interest of the individual, whereas others-for example, a severe reaction to the first injection-remain valid. Perhaps this factor influenced many of the respondents who thought that the current government recommendations as they are reflected in the list of questions should be ignored.

At first sight the contraindications read well enough, and to those not familiar with current practice they look sensible and clear. The questionnaire was designed in part to demonstrate some of the difficulties of interpretation. All 22 cases in the questionnaire, it might be argued, were contraindications and indeed there was not one that some respondent did not view in that light. Some, however-for example, 7A and 10B-are in the same class as grandpa's stroke, and had little support. But others were considered by many to be grounds for advising against immunisation. Closer scrutiny of some of these "popular" contraindications leads to more uncomfortable and possibly unanswerable questions. For example, " $12 \mathrm{~B}$, motor delay" is not uncommon in the understimulated child; as such a child from a deprived background is more likely to have experienced a troubled perinatal period, underlying brain damage may well be difficult to exclude; likewise his risk of contracting and suffering badly from whooping cough is also high. Yet many

What health hazards are encountered in the preparation of hides for shoe making, and what risks are there in the glues used in the process? What protective measures are advised for the workers?

In the shoe industry workers may be exposed to physical and chemical hazards. The main physical hazard is the noise of cutting, stitching, and sewing machines. Vibration transmitted to the fingers by pressing soles against the pounding roller can produce Raynaud's phenomenon. Nevertheless, the main hazards in this industry are chemical and linked to the use of glues and polishes. The degree of exposure to inhalation and the nature of adverse effects depend on the volatility and toxicity of solvents. The stitching and lasting processes that comprise the preparation of the upper section of shoes are the combination of sewing and cementing. The solvent here is mostly benzene. In the stock-fitting process, when the bottom part of the shoe is manufactured, the main solvents are trichloroethylene, carbon would consider that protection is not recommended. True, in practice, the other factors could be taken into account and they might make the decision to immunise easier; but how often would that be the case?

Three situations may be defined: (a) those in which immunisation should not be given-for example, after a previous severe systemic reaction; (b) those in which immunisation should be given only under very exceptional circumstances-for example, during a febrile illness or when a previous injection caused a severe local reaction; and $(c)$ those in which it is appreciated that the child might be at an increased risk of having a central nervous system reaction where the balance of risk is considered-for example, a history of illness that might have led to temporary or permanent brain injury.

Although the prime consideration of all those concerned in drawing up or interpreting the contraindications is for the protection and benefit of the child, other influences are bound to operate. For example, government preventive health policies depend on public confidence and therefore official advice is likely to err towards overcaution and inaction. Manufacturers, while wishing to promote their product, will also seek to avoid the financial consequences of any damage its use might cause. Doctors and health visitors must consider their own position; recommending or giving immunisation to children in the face of what might be judged to be an officially stated contraindication clearly brings with it the possibility of being found to be negligent. This might explain the differences in the answers of the givers as opposed to the advisers in the survey. The answers to the questionnaire show that in many cases, for every expert who would advise against there is another who would take the opposite view. It is inevitable that, consciously or not, doctors and health visitors will be reluctant to recommend immunisation if they fear that the child might already have brain damage and there is the possibility that the vaccine and they in recommending it might be later held responsible for causing or contributing to the handicap that only subsequently became apparent. This concern needs to be removed if there is a genuine desire to increase the rate of whooping cough immunisation.

I am grateful for the help given by Dr Malcolm Lewis, Dr Leon Polnay, Dr Eleanor More, Dr Nick Spencer, Dr Marion Jepson, Dr W O Williams, and Dr Mike Sheldon and to everyone who completed the questionnaire.

\section{References}

1 Miller DL, Ross EM, Alderslade R, Bellman MH, Rawson NSB. Pertussis immunisation and serious acute neurological illness in children. $\mathrm{Br}$ Med F $1981 ; 282: 1595-9$

2 Department of Health and Social Security. Whooping cough: reports from the Committee on Safety of Medicines and the foint Committee on Vaccination and Immunisation. London: HMSO, 1981.

(Accepted 28 August 1981)

tetrachloride, acetone, and alcohols. The same applies to bottoming and heeling.

The basic protective measure is directed to decreasing the concentration of solvents in the working atmosphere. The cheapest method is to keep every container closed when not in use and to fill the working containers from the larger ones outside the work shop. These precautions may be adequate in not-crowded work shops with good natural ventilation, but in many cases the installation of mechanical ventilation, possibly with local exhaust ventilation, is unavoidable. As exposure is continuous, the use of respirators is not practicable. Glues or polishes may contain irritants and sensitisers and therefore protective gloves resistant to solvents are necessary. The frequency of periodical medical examination depends on the type of solvents. Symptoms such as headache, fatigue, dizziness, vertigo, and nausea are signals of overexposure and may occur independently of the type of solvents used. 